\title{
ALTERACIÓN QUÍMICA DE LA SOLUCIÓN DE UN SUELO SULFATADO ÁCIDO, CON ENCALAMIENTO Y LAVADO EN COLUMNA DISTURBADAS
}

\section{ALTERATION OF ACID SULPHATE SOIL SOLUTION CHEMISTRY WITH LIMING AND WASHING IN DISTURBED COLUMNS}

\author{
Enrique Combatt Caballero ${ }^{1}$ \\ Teobaldis Mercado Fernández ${ }^{2}$ \\ Graciliano Palencia Severiche ${ }^{3}$
}

\section{RESUMEN}

La acidez de los suelos es uno de los principales factores que limitan la producción de los suelos sulfatados ácidos tropicales, siendo lenta la corrección con cal agrícola y lavado. El trabajo buscó evaluar el efecto de encalamiento y lavado sobre las características químicas: $\mathrm{pH}$, conductividad eléctrica y contenidos de sulfatos, calcio y magnesio en los lixiviados de un suelo sulfatado ácido (isohipertermico typic sulfaquepts), en San Carlos, Córdoba, Colombia. Fueron instalados lisímetros de $15 \mathrm{~cm}$ de diámetro $\mathrm{x} 80 \mathrm{~cm}$ de altura, rellenados con suelo muestreado en los horizontes superficiales (0-20 y $20-40 \mathrm{~cm}$ ), corregidas con dosis de cal de 0, 2, 4, 6, 8 y 10t/ha y lavado con 15L de agua, equivalentes a tres volúmenes de poros. Se utilizó un diseño completamente al azar con cuatro repeticiones, en casa de vegetación de la Universidad de Córdoba. Las variables evaluadas fueron $\mathrm{pH}$, conductividad eléctrica (CE), sulfatos, calcio y magnesio en los lixiviados. El encalamiento aumentó poco el $\mathrm{pH}$; de igual forma, se encontró reducción en la CE y los tratamientos de cal y lavado no incrementaron la lixiviación de sulfatos, calcio y magnesio. Fueron

${ }^{1}$ I.A., M.Sc. Docente Facultad de Ciencias Agrícolas. Universidad de Córdoba. ecombatt@sinu.unicordoba.edu.co

2 I.A., M.Sc. Docente Facultad de Ciencias Agrícolas. Universidad de Córdoba.

${ }^{3}$ I.A. Esp. En suelos y aguas. Docente Facultad de Ciencias Agrícolas. Universidad de Córdoba. obtenidas ecuaciones de regresión que permitieron estimar, aproximadamente, 10t/ha de cal, para elevar el $\mathrm{pH}$ a 5 y reducir la $\mathrm{CE}$ a $2 \mathrm{dS} / \mathrm{m}$.

Palabras clave: Cal agrícola, lixiviación, $\mathrm{pH}$, conductividad eléctrica, sulfatos, calcio, magnesio.

\section{SUMMARY}

Soil acidity is on of the main factors which limit production of tropical acid sulfate soils, being the correction with liming and washing slow. The aim of this research was to evaluate the effect of liming and washing on the chemical characteristics, $\mathrm{pH}$, electric conductivity, sulfates, calcium and magnesium content of lixiviates in an acid sulfate soil (franc isohipertermico typic sulfaquepts) from San Carlos, Córdoba, Colombia. Lysimeters, $15 \mathrm{~cm}$ of diameter $\mathrm{x} 80 \mathrm{~cm}$ of height were installed, and filled with soil collected from the surface horizon, $0-20$ and $20-40 \mathrm{~cm}$, limed with 0, 2, 4, 6, 8 and $10 \mathrm{t} / \mathrm{ha}$, and washed with $15 \mathrm{~L}$ of water, equivalent to three pore volumens. The research was carried out with a randomized block design with four replications, in a hothouse of the University of Córdoba, Colombia. The evaluated variables were $\mathrm{pH}$, electrical conductivity (EC), sulfates, calcium and magnesium contents in the leaching. A small increase in $\mathrm{pH}$ due to liming was detected, as well as a reduction in EC; however, liming and washing did not increase the lixivation of sulfates, calcium and magnesium. Regressions models were obtained, which permitted to, approximately, estimate $10 \mathrm{t} / \mathrm{ha}$ lime to increase $\mathrm{pH}$ to 5 and to reduce EC to $2 \mathrm{dS} / \mathrm{m}$. 
Key words: Lime, lixiviation, $\mathrm{pH}$, electric conductivity, sulfates, calcium, magnesium.

\section{INTRODUCCIÓN}

En las zonas de bacines del río Sinú (Córdoba), se están extendiendo las áreas de suelos sulfatados ácidos (SSA), como resultado de la implementación de redes de drenaje para la actividad agropecuaria, lo cual, contribuye como una fuente proveedora de metales y de compuestos ácidos que afectan el ambiente edafológico. En estas condiciones, se presentan cambios en la reacción del suelo $(\mathrm{pH})$, por los constantes cambios en los procesos de óxido-reducción, que son donantes de protón $\left(\mathrm{H}^{+}\right)$, que acidifican los suelos. En SSA, estos protones se pueden derivar del ácido sulfúrico libre, de sulfuros de hidrógenos y de la desorción del sulfato adsorbido, que favorecen la hidroxilación del $\mathrm{Al}$ y Fe y estos, a su vez, producen hidrogeniones, que aumentan la acidificación en estas zonas (Bennett et al. 2004).

Esta acidificación reduce considerablemente la productividad agrícola, lo que se ve reflejado en el estrés fisiológico de los cultivos, debido a que el ácido soluble, entre otros efectos, desciende el $\mathrm{pH}$, a tal punto que, las altas concentraciones de iones hidrógeno en la solución del suelo, promueven el ataque a las estructuras cristalinas de las arcillas, lo que permite que se liberen iones de aluminio y de hierro a la solución (Sammut, 2002).

Los metales pesados Fe y Al junto a las altas cantidades de acidez generada por la oxidación de la pirita afectan las características del suelo y del ambiente (Shamshuddin et al. 2004). Según MacDonald et al. (2004), la oxidación de la pirita puede llevar a degradación medioambiental severa, como muertes de plantas, declives drásticos en la calidad de agua a través de la exportación de drenaje ácido y de metales disueltos, y muertes de organismo que existen en aguas receptoras.

En Colombia, los SSA se presentan en los departamentos de Sucre, Bolívar, Boyacá, Valle del Cauca y Córdoba; en este último departamento existen entre 10.000 e 20.000ha, donde el drenaje y el secamiento de las zonas de bacines de la llanura aluvial dirige los procesos hacia la acidificación y la formación de suelos sulfatados ácidos (Combatt et al. 2004).
La corrección de la acidez que se origina en estos suelos envuelve la neutralización de la acidez con lavado y enmiendas agrícolas, particularmente, cal agrícolas. El sistema funciona, porque entre otros procesos químicos que se presentan en estos suelos, se promueve la unión de iones que originan la acidez $\left(\mathrm{H}^{+}, \mathrm{Al}, \mathrm{Fe}\right)$ con los iones hidroxilos, que se producen por la hidrólisis del carbonato de calcio o magnesio, en condiciones adecuadas de humedad del suelo, con la consecuente neutralización de elementos acidificantes, por precipitación de compuestos insolubles. Además, con las láminas de lavado, se logra la exportación de mucha acidez, como también de los iones y compuestos que promueven la oxidación de sulfuros, que es la fuente esencial de generación de ácidos y principal factor limitante en las producciones agropecuarias en SSA.

El actual conocimiento y entendimiento del manejo de los SSA interiores es incompleto, muy poco conocido y con deficientes estudios científicos, necesitando desarrollar estrategias que desminuyan los impactos negativos en los ecosistemas que posean características de alta acidez, originada por la oxidación de las diferentes forma de sulfatos.

En el manejo de esta problemática, a nivel mundial y nacional, diversos autores señalan que la acidez puede ser corregida mediante el encalado, neutralizando los iones hidrógeno de la solución del suelo con los iones hidroxilo, que se producen al aplicar los materiales encalantes (Ward et al. 2002). El carbonato de calcio, se disuelve a medida que los iones hidroxilo son removidos de la solución del suelo, produciendo iones calcio y bicarbonato. Mensvoort et al. (1991) y Cook et al. (2000) indican que la acidez soluble e intercambiable debe ser removida tanto como sea posible antes de aplicar enmiendas y explican que, en principio, la lixiviación con agua dulce es eficiente en la remoción del suelo de $\mathrm{H}^{+}$y de $\mathrm{H}_{2} \mathrm{SO}_{4}$, como también de iones metales que producen $\mathrm{H}^{+}$por reacciones de hidrólisis y oxidación, como son las sales solubles de Fe y Al del suelo.

Mongia et al. (1997) exponen que el uso de cal agrícola disminuyó el contenido de Al del suelo ácido y redujo el nivel de toxicidad en suelos sumergidos, alternativamente sumergidos y saturados, después de 30 días de incubación. De igual forma, parte del contenido de Al intercambiable, se neutralizó a través del período de la incubación, cuando se aplicó cal en suelos sumergidos. 
Otros autores expresan que la práctica del encalamiento es a menudo cara y poco eficiente en muchas partes de los trópicos (Sierra et al. 2003) y recomiendan realizar lavados antes de encalar y, posteriormente, fertilizar (Mathew et al. 2001).

Reflexionando sobre el área creciente de SSA en el bajo Sinú (Córdoba), se determinó evaluar el efecto de encalamiento y lavado, en las características químicas de $\mathrm{pH}$, conductividad eléctrica, y la cantidad de calcio, magnesio y sulfatos en los lixiviados, de un suelo sulfatado ácido de Córdoba.

\section{MATERIALES Y MÉTODOS}

El estudio, se ejecutó en casa de vegetación de la Universidad de Córdoba, (Montería - Colombia), ubicada a $8^{\circ} 75^{\prime}$ de latitud Norte y $75^{\circ} 55^{\prime}$ de longitud Oeste, a una altura de $15 \mathrm{msnm}$, HR del $85 \%$ y temperatura promedio $28^{\circ} \mathrm{C}$. El experimento, se realizó en laboratorio con columnas de percolación, se utilizó un suelo sulfatado ácido (franco mixto isohipertermico typic sulfaquepts), localizado en el municipio de San Carlos, Córdoba, Colombia, en el valle del río Sinú. Las muestras de suelo fueron colectadas separadamente en el primero y segundo horizonte $(0-20 \mathrm{~cm}$ y $20-40 \mathrm{~cm})$, llevadas al laboratorio secadas al aire y tamizadas por malla de $2 \mathrm{~mm}$.

Las unidades experimentales fueron constituidas por columnas de lixiviación, confeccionadas a partir de tubos de cloruro de polivinilo de $15,48 \mathrm{~cm}$ de diámetro por $80 \mathrm{~cm}$ de largo, las cuales, se llenaron con suelo hasta los $40 \mathrm{~cm}$, tratando de mantener la secuencia encontrada en campo de los primeros $40 \mathrm{~cm}$ del perfil. Las columnas, se sellaron en la parte inferior con tela plástica de $1 \mathrm{~mm}$ de abertura, a fin de evitar la eluviación del suelo y debajo de la malla papel filtro (Whatman No 42) y malla plástica, nuevamente, para evitar la salida del suelo con el efluente. En cada columna, se colocaron embudos de siete pulgadas de diámetro para direccionar y captar los lixiviados en frasco de colecta. Los $40 \mathrm{~cm}$ de la parte superior no fueron rellenados con suelo, a fin de facilitar la adición de agua a medida que pasaban los volúmenes porosos iniciales.

Los tratamientos, correspondientes a las dosis de cal, se incorporaron en el primer horizonte, equivalentes a 0,1 , 2, 4, 6, 8 y 10t/ha de cal agrícola comercial. Las columnas fueron acondicionadas verticalmente en soportes de madera, cuya base quedó $20 \mathrm{~cm}$ encima de la superficie, para dejar un espacio y poder colectar cada $500 \mathrm{~mL}$ de solución lixiviada. Las columnas fueron encubadas por 30 días, manteniéndose la humedad del suelo próxima a la capacidad de campo y después fueron aplicados tres volúmenes de poros, equivalentes a 15L/columna de agua de lluvia. Se empleó un diseño experimental completamente al azar, con cuatro repeticiones.

Las variables evaluadas fueron $\mathrm{pH}$, conductividad eléctrica $(\mathrm{CE})$, calcio (Ca), magnesio $(\mathrm{Mg})$ y sulfatos $\left(\mathrm{SO}_{4}{ }^{2-}\right)$ en los lixiviados. Los análisis de suelos, se realizaron de acuerdo con los métodos analíticos recomendados por el Instituto Geográfico Agustín Codazzi (IGAC, 1990):

- $\mathrm{pH}$ : método potenciométrico; relación suelo-agua $1: 1$

- Conductividad eléctrica: pasta de saturación, método potenciométrico

- Calcio y magnesio por espectrofotometría de absorción atómica

- Sulfatos: método cloruro de bario

Al final del estudio y considerando la concentración de $\mathrm{Ca}, \mathrm{Mg}$ y SO 4 en cada volumen lixiviado, se calculó la cantidad lixiviada de estos elementos.

Los resultados fueron sometidos a análisis de variancia, siendo ajustados modelos de regresión para cada variable. Se aplicó el Sistema para Análisis Estadísticas (SAEG). Se escogieron los modelos que poseían los coeficientes de regresión significativos, hasta el nivel de $20 \%$, para así obtener los modelos que mejor explicaran los fenómenos en la dinámica de lixiviación. Los modelos de regresión ajustados a sus coeficientes fueron testados usándose el cuadrado medio del residuo del análisis de variancia.

\section{RESULTADOS Y DISCUSIÓN}

Características químicas iniciales del suelo: En la tabla 1 , se observa que la reacción del suelo es fuertemente ácida en todas las profundidades; el contenido en materia orgánica es alto por las condiciones de reducción dominante en el perfil. El azufre es excesivo, debido a que estas zonas están influidas por materiales de origen que contienen en su estructura gran cantidad de este elemento, al igual que son zonas de bacines, donde ha existido acumulación de diferentes compuestos de sulfatos. 
Tabla 1. Caracterización química y física de un SSA franco mixto isohipertermico typic sulfaquepts antes de la realización del ensayo.

\begin{tabular}{|c|c|c|c|c|}
\hline \multirow{2}{*}{\multicolumn{2}{|c|}{ Características }} & \multicolumn{3}{|c|}{ Profundidad $(\mathrm{cm})$} \\
\hline & & $0-20$ & $20-40$ & $40-70$ \\
\hline \multicolumn{2}{|c|}{ pH em água (1:1) } & 3,8 & 3,7 & 3,77 \\
\hline MO & $(\%)$ & 8,05 & 10,25 & 1,43 \\
\hline S & \multirow{2}{*}{$\mathrm{mg} / \mathrm{kg}$} & 350 & 324,5 & 575,6 \\
\hline$P$ & & 20,5 & 20 & 6,13 \\
\hline $\mathrm{Ca}$ & \multirow{6}{*}{ cmolc $\mathrm{Kg}^{-1}$} & 6,5 & 4,75 & 6,67 \\
\hline $\mathrm{Mg}$ & & 7 & 5,75 & 9,83 \\
\hline K & & 0,55 & 0,2 & 0,10 \\
\hline $\mathrm{Na}$ & & 0,18 & 0,18 & 0,24 \\
\hline $\mathrm{Al}$ & & 8,7 & 6,3 & 11,00 \\
\hline $\mathrm{CIC}$ & & 22,9 & 17,15 & 27,80 \\
\hline $\mathrm{Fe}$ & \multirow{2}{*}{$\mathrm{mg} / \mathrm{kg}$} & 73 & 34,5 & 46,0 \\
\hline $\mathrm{Mn}$ & & 23 & 17,5 & 23,67 \\
\hline & & \multicolumn{3}{|c|}{ Análise granulométrica (\%) } \\
\hline Arena & & 55,15 & 58,05 & 55,5 \\
\hline Limo & & 32,25 & 31,065 & 35,2 \\
\hline Arcilla & & 12,5 & 10,25 & 9,21 \\
\hline \multicolumn{2}{|c|}{ Densidad del suelo $\left(\mathrm{kg} / \mathrm{m}^{3}\right)$} & 1,15 & 1,18 & 1,72 \\
\hline
\end{tabular}

El contenido de fósforo varió de alto a bajo a medida que se profundizó en el perfil; los contenidos de calcio fueron similares en todas las profundidades; el magnesio, se encontró en niveles que excedieron a los del calcio, con porcentajes de saturación mayores del $30 \%$; el contenido de potasio fue alto en la superficie, pero disminuye con la profundidad y, finalmente el contenido de sodio no causa problemas. La acidez intercambiable fue excesiva, lo cual, explica la aparición en los cultivos de síntomas de toxicidad característicos, como atrofiamiento de los sistemas radicales. Con los microelementos, se observó que el hierro posee contenidos altos y el manganeso es alto, pero no excesivo. Se manifiestan porque al encontrarse las zonas de estudio en áreas de bacines, se posibilita la acumulación de altas cantidades de hierro y de manganeso, los cuales, se encuentran en continuo estado de óxido-reducción, lo que trae como consecuencia su incremento.
Variación de la reacción del suelo $(\mathrm{pH})$ con la lixiviación: El ANOVA mostró diferencias altamente significativas, sugiriendo una manifestación diferencial de la reacción del suelo con la aplicación de cal (Tabla 2). Las diferentes dosis de cal empleadas y los 15L de agua promovieron pequeñas alteraciones $(3,26$ a 4,89) en el pH de los lixiviados (Figura 1). Esta mínima diferencia es explicada, posiblemente, por el aumento de hidroxipolímeros de $\mathrm{Al} \mathrm{y,} \mathrm{al} \mathrm{mismo} \mathrm{tiempo,} \mathrm{por} \mathrm{iones}$ hidrogeniones, formados a partir de la disociación ácido sulfhídrico $\left(\mathrm{H}_{2} \mathrm{~S}\right)$, además, de la alta acidez potencial de estos suelos, los cuales evitan un mayor incremento del $\mathrm{pH}$ de la solución lixiviada; esto, probablemente, porque la aplicación de cal agrícola en dosis de hasta 10t/ha, no permitió reducir la acidez, como para evitar la elevada actividad $\mathrm{de}^{+}$, en estas condiciones. Esto tiene su origen debido a que la calcita aplicada como $\mathrm{CaCO}_{3}$ logró reaccionar parcialmente y generar $\mathrm{OH}^{-}$y $\mathrm{HCO}_{3}^{-}$, no 
Tabla 2. Cuadrados medios del análisis de varianza para diferentes variables químicas de un SSA, con diferentes dosis de encalamiento y $15 \mathrm{~L}$ de agua como lavado.

\begin{tabular}{|c|c|c|c|c|c|c|}
\hline $\begin{array}{c}\text { F. DE } \\
\text { VARIACIÓN }\end{array}$ & GL & pH & CE & Ca & Mg & SO $_{4}$ \\
\hline Tratamiento & 5 & $1,45^{* *}$ & $2,46^{* *}$ & $1488,0 \mathrm{~ns}$ & $2668,8^{*}$ & $220180^{* *}$ \\
\hline Residuo & 18 & 0,073 & 0,35 & 1068,7 & 681,7 & 41998 \\
\hline C.V. (\%) & & 7,161 & 18,71 & 24,178 & 20,0 & 14,35 \\
\hline
\end{tabular}

${ }^{*} \mathrm{y} *$ S Significativos al 0,05 y $0,01 \%$, ns = No significativo, respectivamente.

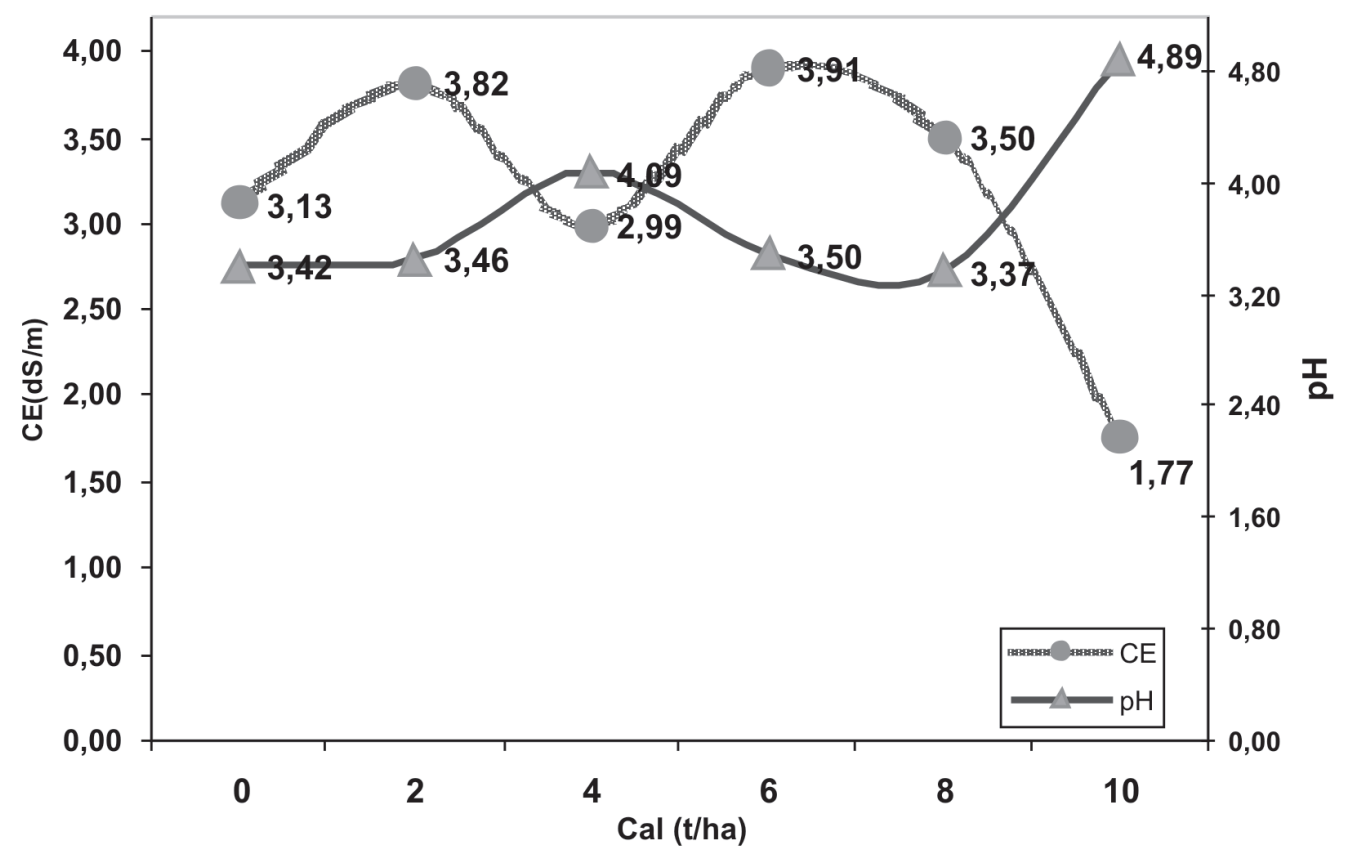

Figura 1. pH y conductividad eléctrica en los lixiviados de un suelo sulfatado ácido, como resultado de dosis de cal y lavado.

suficientes para neutralizar la acción de iones de Al, Fe, $\mathrm{SO}_{4}$, y ácidos libres de la materia orgánica, que generan protones e incrementan la acidez en los lixiviados.

Las condiciones químicas halladas manifiestan que la neutralización de protones en este medio reducido no disminuye la cantidad que es producida por los compuestos ácidos, que al entrar en proceso de hidrólisis, son sustancias que disocian iones $\mathrm{H}^{+}$químicamente. Bennett et al. (2004), exponen que durante la inundación de los suelos sulfato ácidos actuales, minerales de Fe y $\mathrm{Al}$, se pueden disolver, y se liberan iones $\mathrm{H}^{+}$, que se encuentran en la fase intercambiable del suelo.

Palko (1994), percibió que la aplicación de cal en cantidades altas no redujo la acidez en suelos sulfatados ácidos y Weppling (1997), trabajando con altas cantidades de $\mathrm{CaCO}_{3}$ y $\mathrm{CaMg}\left(\mathrm{CO}_{3}\right) 2$ en suelos sulfatados ácidos drenado y sin drenar, halló que la aplicación de cal no fue eficiente para neutralizar la acidez, debido a la altas cantidades de ácidos almacenados en estos suelos ácidos y en el agua. De igual forma, Ponnamperuma 
(1966) citado por Amézquita (1988) constató que el $\mathrm{pH}$ de los SSA, se incrementó de forma lenta y que raramente excede de 6,0, después de seis meses de inundación. Mongia et al. (1998) encontraron que con la aplicación de cal disminuye el contenido de $\mathrm{Al}$ y $\mathrm{Mn}$ soluble e intercambiable, mientras que el Fe aumenta. Por otra parte, Palko (1994) reporta que la aplicación de cal, en forma masiva, no reduce la acidez, para evitar la toxicidad causada por el Al. Para estas condiciones con características químicas estudiadas de SSA que existen en San Carlos, Córdoba es necesario tener presente que la implementación de prácticas de encalamiento de hasta 10t/ha y lavado no logran mejorar sustancialmente las condiciones de $\mathrm{pH}$ de estos suelos, y que su recuperación necesita tiempo y altos costos de inversión. Una posible solución es optar por la no intervención antrópica de estas zonas, dejándolas para otras actividades que requieren menor intervención.

Conductividad eléctrica en los lixiviados: El ANOVA registró diferencias altamente significativas en la conductividad eléctrica, lo que sugiere que la cantidad de cal influyó diferencialmente en el contenido de sales en los lixiviados evaluados (Tabla 2). De forma general, el encalamiento utilizado influenció en la conductividad eléctrica (CE) (Figura 1), ya que de 3,13 se redujo hasta $1,76 \mathrm{dS} / \mathrm{m}$ y esto ocurrió, posiblemente, por que ocurrió solubilización de sales o de compuestos que hacen parte de la fase soluble o intercambiable y, a su vez, un mayor flujo de los iones en la solución lixiviadas que reducen la CE.

La disminución en la CE de los lixiviados indica que la aplicación progresiva de cal y lavado con agua ocasiona la solubilización de compuestos presentes en la fase intercambiable del suelo o de la fase soluble. Estas prácticas aumentan los procesos de disolución de sales que se lixivian e inciden en la reducción de la conductividad eléctrica. Howeler (1973) expone que la conductividad eléctrica aumenta debido a la producción de $\mathrm{Fe}^{2+}, \mathrm{Mn}^{2+}, \mathrm{NH}_{4}^{+}$y después baja por la precipitación del hierro, como $\mathrm{Fe}(\mathrm{OH})_{2}$ y FeS.

Según Sterk (1993), con el agua se remueven a través de los poros y se lixivian rápidamente las sustancias solubles de la interfase agua-suelo y a medida que se incrementa la humedad, decrece la velocidad de infiltración del suelo, lo cual, no contribuye a la remoción efectiva de los elementos tóxicos de la matriz. Estos procesos físico-químicos reducen, sustancialmente, la recuperación de estos suelos, lo que incide en la sostenibilidad de los mismos.

El transporte y el cambio de los solutos en la zona de transición del sistema radical de las especies vegetales es de vital importancia, por que es un estado altamente influenciado por factores, como las propiedades hidráulicas de los sedimentos, como es la infiltración de agua y ésta, a su vez, depende de la porosidad, que es afectada por los procesos de contracción y de expansión de las arcillas; a medida que se presente mayor lixiviación de sales, como sulfato de magnesio y sodio, existirá menor probabilidad de desagregación o desfloculación de la estructura del suelo y, por tanto, una corrección rápida de la propiedades químicas de los SSA. Tobias et al. (2001) documentan que cambios sustanciales en sedimentos salinos son responsables de la variación regional en la entrada de agua en el perfil.

Lixiviación de los sulfatos: El ANOVA reportó diferencias altamente significativas en la concentración de sulfatos en los lixiviados (Tabla 2).

Con relación a las concentraciones de sulfatos (Figura 2) en los lixiviados, estos presentaron disminución en su concentración con el incremento de las dosis de cal y aumento con las láminas de agua aplicada, lo que confirma que juntos son más eficientes para el mejoramiento de las condiciones de alta cantidad de sulfatos de los SSA. Es positivo aplicar cal y aumentar la lámina de lavado de los suelos, para precipitar y lixiviar gran parte de sulfatos y otras fuentes de acidificación, como fue verificado por Van Bremen (1993), quien constató que el método más eficiente para lixiviar la acidez en agua es agregando cal y lavado. En estas condiciones, se aumenta el lavado eficaz del ión sulfato, lo que repercute, positivamente, en el mejoramiento de las propiedades químicas de estos suelos, disminuyendo la posibilidad de volverse a activar la acidez potencial y recuperar las condiciones iniciales de acidificación. Por otra parte, Kashir E Yanful (2000) encontraron que en presencia de carbonato de calcio, la acidez es neutralizada, el hierro precipita y el sulfato se une con el calcio y se forma yeso, que se insolubiliza parcialmente. De igual forma, existe otra posibilidad y es que los sulfatos pueden ser adsorbidos a medida que aumentan los tratamientos, ya que hay intercambio de iones $\mathrm{SO}_{4}^{2-}$ por iones $\mathrm{OH}-$, en la superficie de los óxidos de hierro y de aluminio. Fanning et al. (1998) demuestran que 
después de un tiempo de reducción, el Fe precipita como sulfuro ferroso, por una reacción del sulfuro de hidrógeno con sales de hierro. Drever (1997) verificó que en sedimentos con condiciones de reducción el sulfato, se reduce a sulfito, que puede ser reoxidado y escapar de los sedimentos como $\mathrm{H}_{2} \mathrm{~S}$ o, de igual forma, se puede ligar en los sedimentos como sulfatos de metal insoluble y normalmente el hierro es cuantitativamente el más importante metal, que limita la lixiviación de sulfatos. Smith $\mathcal{E}$ Melville (2004) comprobaron que existe un gradiente negativo en la concentración de $\mathrm{SO}_{4}$ y $\mathrm{Fe}$ en los sedimentos, debido a la formación de monosulfuros de hierro en condiciones de reducción y esto disminuye la acidez y la concentración de metales.

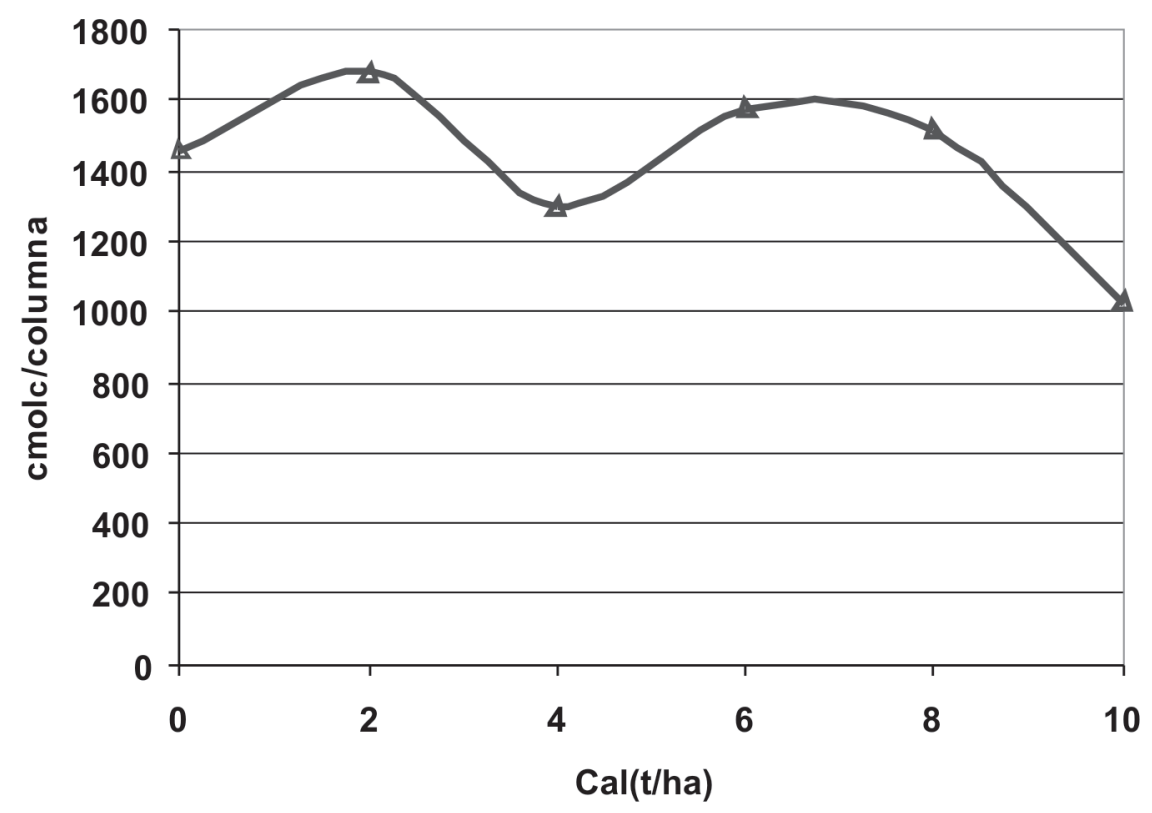

Figura 2. Cantidad de sulfatos en los lixiviados de un suelo sulfatado ácido, como resultado dosis de cal y lavado.

Variación del calcio en los lixiviados: El ANOVA no reportó diferencias significativas en la concentración de calcio en los lixiviados (Tabla 2).

La figura 3 muestra los valores de los contenidos de Ca en los lixiviados; en general, las dosis de cal, mayores de $2 \mathrm{t}$, no proporcionaron grandes incrementos del Ca en solución. Su tendencia fue de mantener casi constante la concentración en los lixiviados, con una pequeña disminución a los 15L de agua lixiviados. Esta reducción de $\mathrm{Ca}$ intercambiable y del $\mathrm{Ca}$ que hace parte del carbonato de calcio, es consecuencia del proceso de adsorción del ión calcio, de la baja solubilidad del carbonato de calcio (Albuquerque et al. 2003) y de la baja permanencia en la solución del suelo de los aniones, adicionados por el encalamiento. Palko (1994) encontró que altas cantidades de cal en un suelo sulfatado ácido sufren una disolución lenta e incompleta del polvo, aplicado como piedra caliza. Watzlaf $\mathcal{E}$ Hedin (1993), aplicando carbonatos de calcio en condiciones de completa reducción, para neutralizar los drenajes ácidos de minas, hallaron poca efectividad, porque los precipitados de ferrihidrita dentro del drenaje, precipitan sobre las partículas de carbonatos de calcio reduciendo su disolución y, por consiguiente, su potencial para la neutralización de la acidez. Por otra parte Golez $\mathcal{E}$ Kyumah (1997), estudiando el efecto de la oxidación de la pirita en algunos nutrientes esenciales, detectaron que lavados repetidos reducen la acidez, pero de igual forma algunos elementos, como $\mathrm{Ca}, \mathrm{Mg}$, $\mathrm{Cu}$ y $\mathrm{Zn}$ son también removidos en la solución.

Variación del magnesio en la lixiviación: El análisis de varianza mostró diferencias significativas en la concentración de magnesio en los lixiviados (Tabla 2). En la figura 3, se muestra que la tendencia del Mg lixiviado 


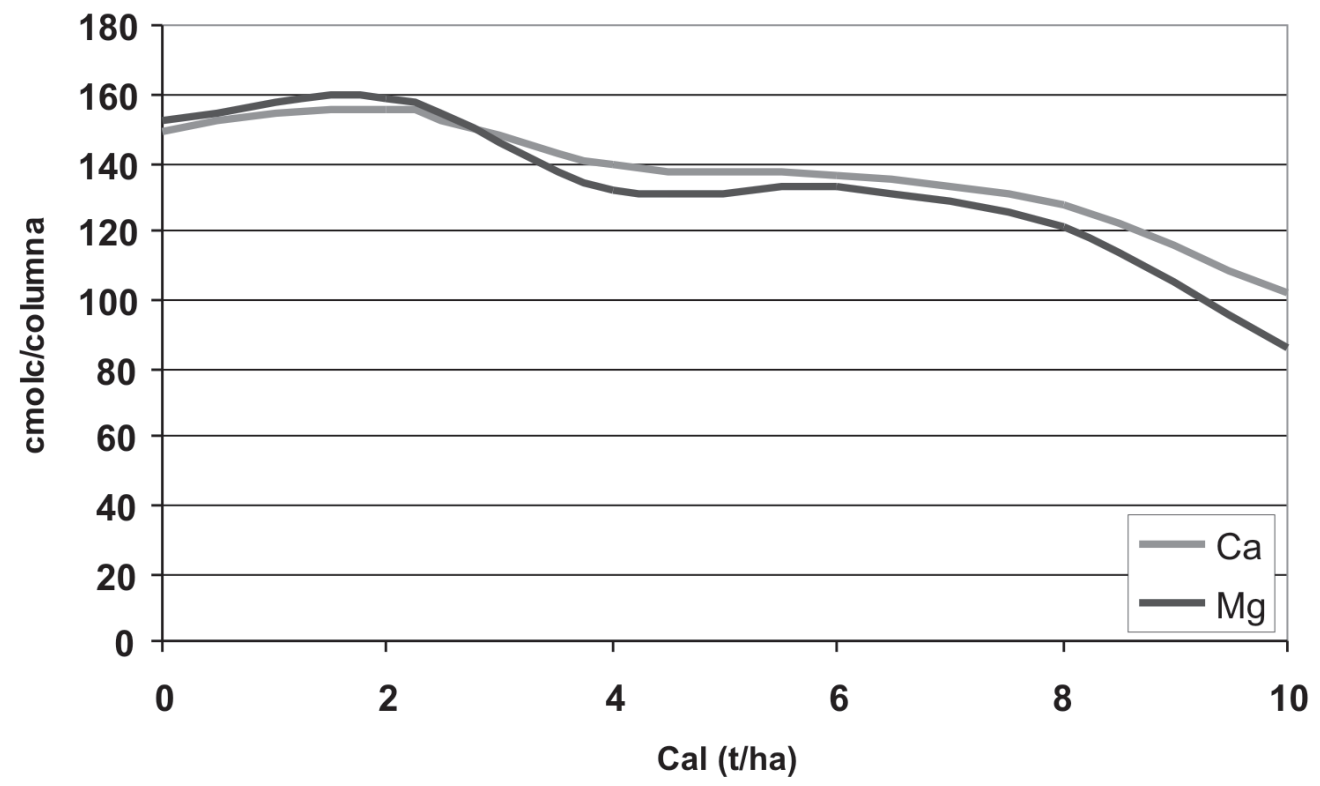

Figura 3. Cantidad de calcio y magnesio en los lixiviados de un suelo sulfatado ácido, como resultado de dosis de cal y lavado.

fue a disminuir a medida que se incrementaron las dosis de cal agrícola, con una tendencia muy similar a la del calcio soluble. Esto, a pesar de ser un elemento de mayor energía de hidratación, que puede ser desplazado con mayor facilidad en los lixiviados, hecho no observado en esta investigación. Esto no coincide con lo expuesto por Sammut et al. (1996), quienes afirman que los sulfuros en sedimentos oxidados forman ácido sulfúrico, y estos ácidos reaccionan con los minerales de las arcillas, liberan sílice y principalmente iones metales como Al, $\mathrm{Fe}$, potasio, sodio y magnesio.

Así mismo, estas prácticas de encalamiento y lavado ocasionan una leve tendencia a aumentar la reacción de suelo $(\mathrm{pH})$, lo que sin duda alguna puede originar cargas dependientes del $\mathrm{pH}$, que aumentan la adsorción de ciertos elementos químicos de mayor valencia y menor radio de hidratación. Albuquerque et al. (2003) exponen que este aumento de cargas negativas puede disminuir la lixiviación de calcio y magnesio.

Como práctica de mejoramientos de las condiciones químicas de aquellas zonas que se desean someter a la actividad agropecuaria es necesario implementar, básicamente, la práctica de lavado empleando una red de drenaje adecuada, suficiente para evacuar el exceso agua $\mathrm{y}$, de esta forma, ir recuperando estas zonas. Paralelo a esto, se pueden sembrar especies tolerantes a la alta acidez, con el fin de buscar un beneficio económico y poder entrar con la estrategia del encalamiento.

Modelos de regresión ajustados: Los valores de $\mathrm{pH}$, de conductividad eléctrica, de sulfatos, de calcio y de magnesio permiten estimar las dosis de cal agrícola necesaria para conseguir el $\mathrm{pH}$ y la conductividad eléctrica deseada y monitorear las cantidades de sulfato, de calcio y de magnesio, que se pueden lixiviar con una dosis de cal agrícola determinada. Todas las modificaciones pueden ser comparadas para los volúmenes de agua lixiviada y estimar la cantidad de cal que se necesita para conseguir una determinada condición química, que son informaciones importantes (Tabla 3).

En esta tabla, se observa que con el uso de estas ecuaciones, se estimó una dosis de 10,2t/ha de cal para aumentar el $\mathrm{pH}$ a 5,0, utilizando $15 \mathrm{~L}$ de agua, y para reducir la conductividad eléctrica a $2 \mathrm{dS} / \mathrm{m}$, se necesitan 9,8t/ha de cal y también la cantidad de sulfatos, de calcio y de magnesio que se lixivia con la aplicación de $10 \mathrm{t} /$ ha de cal. 
Tabla 3. Ecuaciones estimadas de pH (1:1), CE (dS/m) y contenido de $\mathrm{SO}_{4}^{2-}$, Ca y $\mathrm{Mg}$ (cmolc/columna) lixiviados en suelo sulfatados ácidos, en función de dosis de cal (t/ha).

\begin{tabular}{|c|c|c|c|}
\hline $\begin{array}{l}\text { Cantidad de } \\
\text { agua }\end{array}$ & $\begin{array}{l}\text { Ecuaciones } \\
\text { (pH) }\end{array}$ & R2 & Para obtener un $\mathrm{pH}=5,0$ \\
\hline \multirow[t]{2}{*}{$15 \mathrm{~L}$} & $Y \quad \hat{y}=3,2954+0,56025^{\star * *} x-0,161833^{* * *} x^{2}+0,012088^{* * *} x^{3}$ & 0,78 & 10,2 t/ha de Cal \\
\hline & (CE) & & Para una CE de 2dS/m \\
\hline \multirow[t]{2}{*}{$15 \mathrm{~L}$} & $\hat{y}=3,2800-0,154742 x+0,096961^{00} x^{2}-0,0095839^{\circ} x^{3}$ & 0,76 & $9,8 \mathrm{t} / \mathrm{ha}$ de Cal \\
\hline & $\left(\mathrm{SO}_{4}^{2-}\right) \mathrm{cmol} / \mathrm{c}$ Columna & & \\
\hline \multirow[t]{2}{*}{$15 \mathrm{~L}$} & $\hat{y}=1521,66-66,0208 x+26,3129 x^{* 2}-0,024291^{00} x^{3}$ & 0,60 & $3468,45^{(1)}$ \\
\hline & $\left(\mathrm{Ca}^{2+}\right) \mathrm{cmol}_{\mathrm{c}} / \mathrm{Columna}$ & & \\
\hline \multirow[t]{2}{*}{$15 \mathrm{~L}$} & $\hat{y}=158,671-4,69206^{*} X$ & 0,82 & $111,7504(1)$ \\
\hline & $\left(\mathrm{Mg}^{2+}\right) \mathrm{cmol}_{\mathrm{c}} /$ Columna & & \\
\hline $15 \mathrm{~L}$ & $\hat{y}=162,157-6,32413^{* * *} X$ & 0,83 & $98,9157^{(1)}$ \\
\hline
\end{tabular}

(1) Con 10 t/ha de cal cantidad de $\mathrm{cmol}_{\mathrm{c}}$ de $\mathrm{SO}_{4}^{2-}, \mathrm{Ca}^{2+} \mathrm{y} \mathrm{Mg}^{2+}$ lixiviado por columna

*** significativo $0,1 \%$; ** $1 \% ;{ }^{*} 5 \% ;{ }^{0} 10 \% ; 0020 \%$

\section{CONCLUSIONES}

El encalamiento y lavado de un suelo sulfatado ácido de San Carlos, Córdoba no elevaron significativamente el $\mathrm{pH}$ de los lixiviados en columnas disturbadas.

La conductividad eléctrica se redujo significativamente por las dosis de cal agrícola y el lavado, indicando que hubo lixiviación y precipitación de aniones y cationes.

Los análisis de los resultados demuestran que se presentó una alta disminución de los sulfatos lixiviados, a medida que se aumentaron las dosis de cal agrícola. Las dosis de cal agrícola no influenciaron en el aumento del calcio y el magnesio lixiviado.

Se recomienda realizar investigaciones de campo utilizando piezómetros y analizar las características de los lixiviados colectados.

\section{BIBLIOGRAFÍA}

ALBUQUERQUE, J.; BAYER, C.; ERNANI, P.; MAFRA, A.; FONTANA, E. 2003. Aplicação de calcário e fósforo e estabilidade da estrutura de um solo ácido. Rev.. Bras. Cienc. Solo. 27:799-806.
AMEZQUITA, E. 1988. Establecimiento de las zonas oxidadas y reducidas en suelos sumergidos. En: Sociedad Colombiana de la Ciencia del Suelo. Primer seminario de azufre. Colombia, Cali, p.39-41, 43.

BENNETT, C.; WHITE, I.; KEENE, A.; MELVILLE, M.; REYNOLDS, J. 2004. Acidity, metals and acid sulfate soils. Disponible desde Internet en: http:// www.regional.org.au/au/asssi/supersoil2004/s1/ oral/1797 macdonald.htm\#TopOfPage (con acceso 05/07/2008).

COMBATT, E.; GARCÍA, A.; MERCADO, F. 2004. Efecto del encalamiento y el lavado sobre algunas propiedades químicas de un suelo sulfatado ácido magnésico del valle del rió Sinú. Tesis de magíster en suelos. Universidad de Córdoba, Colombia. $167 p$.

COOK, F.; HICKS, W.; GARDNER, E.; CARLIN, D.; FROGGATT, D.W. 2000. Export of Acidity in Drainage Water from Acid Sulphate Soils. Marine Pollution Bulletin. 41(7-12):319-326.

DREVER, J.I., 1997. The Geochemistry of NaturalWaters. Surface and Groundwater Environments. Prentice Hall, New York. 436p. 
FANNING, S.; BURCH, N.; AUERSWALD, K.; BIGHAM, J. 1998. Acid sulphate soils and some associated environmental problems. Advances in Geoecology. 30:145-158.

GOLEZ, N; KYUMAH, K. 1997. Influence of pyrite oxidation and soil acidification on some essential nutrient elements. Aquacultural Engineering. 16:107-124.

HOWELER, R.H. 1973. La química de suelos inundados. CIAT. Cali Colombia. 5p.

IGAC. 1990. Métodos analíticos del laboratorio de suelos. Bogotá. $5^{\mathrm{a}}$. Ed.

KASHIR, M.; YANFUL, E. 2000. Compatibility of slurry wall backfill soils with acid mine drainage. Adv. Environm. Res. 4:252-268.

MacDONALD, B.C.T.; SMITH, J.; KEENE, A.F.; TUNKS, M.; KINSELA, A.; WHITE, I. 2004. Impacts of runoff from sulfuric soils on sedimentary chemistry in an estuarine lake. Sc. Total Environm. 329:115130.

MATHEW, E.; PANDA, R.; NAIR, M. 2001. Influence of subsurface drainage on crop production and soil quality in a low-lying acid sulphate soil. Agric. Water Managem. 47:191-209.

MENSVOORT, M.E.F. VAN.; DUANG VAN NI.; VAN DER SCHANS, J. 1991. Improvement of acid sulphate soils by leaching with salt or brackish water. In: Deturck, P.; Ponnamperuma, F.N. (eds.). Rice production on acid soils of the tropics. Inst. Fundamental Studies, Kandy. p.219-224.

MONGIA, A.; SINGH, N.; MANDAL, L.; GUHA, A. 1997. Effect of soil water regimes and liming on chemical changes and nutrient transformations in acid and acid sulphate soils. J. Indian Soc. Soil Scie. 45(2):344-348.

MONGIA, A.; SINGH, T; MANDAL, L.; GUHA, A. 1998. Effect of lime and phosphorus application on nutrient transformations in acid and acid sulphate soils under submergence. J. Indian Soc. Soil Scie. 46(1):18-22.
PALKO, J. 1994. Lime requirement experiments in acid sulphate soils. Soil-and-Plant-Science. 44(3):149156.

SAMMUT, J.; WHITE, I.; MELVILLE, M. 1996. Acidification of an estuarine tributary in eastern Australia due to drainage of acid sulfate soils. Marine and Freshwater Res. 47:669-684.

SAMMUT, J. 2002. An introduction to acid sulfate soils NSW Agriculture. NSW. School of Geography. Disponible desde Internet en: http://www.agric.nsw. gov.au/reader/ 10742. (con acceso 24/08/04).

SHAMSHUDDIN, J.; MUHRIZAL, S.; FAUZIAH, I.; VAN RANST, E. 2004. A laboratory study on pyrite oxidation in acid sulfate soils. Commun. Soil Sci. Plant Anal. 35(1,2):117-129.

SIERRA, J.; NOEL, C.; DUFOUR, L.; OZIER-LAFONTAINE, H.; WELCKER, C.; DESFONTAINES, L. 2003. Mineral nutrition and growth of tropical maize as affected by soil acidity. Plant Soil. 252:215-226.

SMITH, J.; MELVILLE, M. 2004. Iron monosulfide formation and oxidation in drain-bottom sediments of an acid sulfate soil environment. Applied Geochemistry. 19:1837-1853.

STERK, G. 1993. Leaching of acid from the top soil of raised beds on acid sulphate soil in the Mekong delta, Vietnam. In: Dent, D.L.; van Mensvoort, M.E.F. (Edit.), Selected papers on the Ho Chi Minh City Symposium on Acid Sulphate Soils. Publication No. 53, p.241-246.

TOBIAS, C.; HARVEY, J.; ANDERSON, I. 2001. Quantifying groundwater discharge through fringing wetlands: seasonal variability, methods comparison and implications for wetland-estuary exchange. Limnol. Oceanogr. 46:604-615.

VAN BREMEN. 1993. Environmental aspects of acid sulphate soils. Ho-Chi Minh City, Vietnam, March, No.391- 402.

WARD, N.; SULLIVAN, L.; BUSH, R. 2002. Sulfide oxidation and acidification of acid sulfate soil materials treated with $\mathrm{CaCO}_{3}$ and seawater- 
neutralized refinery residue. Austr. J. Soil Res. 40:1057-1067.

WATZLAF, G.; HEDIN, R. 1993. A method for predicting the alkalinity generated by anoxic limestone drains. In: Proceedings $14^{\text {th }}$ Annual West Virginia Surface Mine Drainage Task Force Symposium. (Morgantown, West Virginia). p.1-12.
WEPPLING, K. 1997. On the assessment of feasible liming strategies for acid sulphate waters in Finland. Dissertationes geographicae Universitatis Tartuensis, 5:81p.

Recibido: Agosto 5 de 2008

Aceptado: Febrero 7 de 2009 